\title{
Mucormycosis infection in severe COVID-19 patient with multiple underlying health conditions
}

\author{
Zahra Heydarifard $^{1} \odot$ | Moslem Safaei $^{2}$ | Sevrin Zadheidar ${ }^{1}$ | Soroush Ehsan $^{3}$ | \\ Nazanin Zahra Shafiei-Jandaghi ${ }^{1}$
}

\author{
${ }^{1}$ Virology Department, School of Public \\ Health, Tehran University of Medical \\ Sciences, Tehran, Iran \\ ${ }^{2}$ Department of Pharmacy, School of \\ Pharmacy, Shahid Sadoughi University \\ of Medical Science, Yazd, Iran \\ ${ }^{3}$ Department of Neurology, Valiasr Naja \\ Hospital, Shahid Beheshti University of \\ Medical Sciences, Tehran, Iran

\section{Correspondence} \\ Nazanin Zahra Shafiei-Jandaghi, \\ Virology Department, School of Public \\ Health, Tehran University of Medical \\ Sciences, Tehran, Iran. \\ Email: nz-shafiei@tums.ac.ir

\section{Funding information} \\ The authors received no financial \\ support for the research, authorship, \\ and/or publication of this article
}

\author{
Abstract \\ Patients with underlying health conditions are vulnerable to invasive fungal in- \\ fection following COVID-19. It seems that uncontrolled diabetes mellitus makes \\ patients susceptible to both severe COVID-19 and mucormycosis. \\ K E Y W O R D S \\ mucormycosis, SARS-CoV-2, severe COVID-19, underlying health conditions
}

\section{1 | INTRODUCTION}

Coronavirus disease 2019 (COVID-19) is a fearsome infectious disease with several complications. This is a case of acute invasive rhino-orbital mucormycosis in a 62-year-old man with severe COVID-19 pneumonia who had a history of uncontrolled diabetes mellitus along with ketoacidosis and nephropathy.

Coronavirus disease 2019 (COVID-19) has infected more than 180 million people across the world and over 3 million in Iran. ${ }^{1}$ SARS-CoV-2 can cause severe infections in patients who have underlying health conditions. ${ }^{2}$

It was suggested that SARS-CoV-2 might increase the susceptibility to pulmonary fungal co-infections. ${ }^{3}$
Mucormycosis is an angioinvasive disease caused by a fungus of the order Mucorales like Rhizopus, Mucor, Rhizomucor, Cunninghamella, and Absidia. ${ }^{4}$

The prevalence of mucormycosis has been 29.7 cases/ year in past six years in Iran. ${ }^{5}$ It is a rare infection with a high mortality rate (20\%-100\%). Immunocompromised patients such as those with hematological malignancies, recipients of hematopoietic stem cells (HSCT), and patients with diabetes mellitus and ketoacidosis are susceptible to the highly fatal mucormycosis infection. ${ }^{6,7}$

While super-infections and co-infections in COVID-19 pneumonia are still under investigation, sporadic reports of mucormycosis in COVID-19 patients have been published. 
Herein, we present a case of severe COVID-19 with multiple underlying health conditions who was coinfected with rhino-orbital mucormycosis.

\section{2 | CASE PRESENTATION}

A 62-year-old man with a past medical history of type 2 diabetes mellitus, hypertension, and diabetic nephropathy under hemodialysis presented to the Emergency Department of Valiasr Naja Hospital, Tehran, Iran, on April 29, 2021, with shortness of breath and hypoxia. He had a history of coronary artery bypass grafting (CABG) and cataract surgery for both eyes. On admission, he had a temperature of $37.3^{\circ} \mathrm{C}$, heart rate of 100 , respiratory rate of 24, blood pressure of $117 / 63 \mathrm{~mm} \mathrm{Hg}$, and $\mathrm{O}_{2}$ saturation of $60 \%$. Moreover, crackle sounds were heard on auscultation. Upon examination, he was diagnosed with acute respiratory distress syndrome (ARDS); therefore, he was admitted to the intensive care unit (ICU) for 10 days. On day 8 of hospitalization, the patient complained of a headache and pain in the right eye followed by a more severe headache and edema in the right eye on the next day.

On day 10 of hospitalization, his health condition exacerbated. He had vision loss in the right eye and proptosis in both eyes. Ocular examination showed chemosis, conjunctivitis, and cherry red spot in the right eye, indicating central retinal artery occlusion (CRAO).

He was transferred to an ophthalmology center (Rasoul Akram Hospital) for endoscopic surgical debridement. Unfortunately, he expired on day 30 of admission.

\section{1 | Investigations}

The results of blood chemistry, complete blood count (CBC), and blood gas tests are shown in Table 1. The patient had a high WBC count of $11,300 \mathrm{~mm}^{3}$ with 91\% neutrophils and 7\% lymphocytes. The results of other hematological tests were as follows: blood sugar $291 \mathrm{mg} / \mathrm{dl}$, bicarbonate $17.8 \mathrm{mEq} / \mathrm{L}$, creatinine $5.4 \mathrm{mg} /$ dl, BUN $46.7 \mathrm{mg} / \mathrm{dl}$, and lactate dehydrogenase $580 \mathrm{U} / \mathrm{L}$. Laboratory investigations revealed nephropathy and hyperglycemia with diabetic ketoacidosis (DKA).

Reverse transcription-polymerase chain reaction (RTPCR) was positive for COVID-19. A chest computed tomography (CT) was performed, which revealed bilateral peripheral ground-glass opacification with extensive lung parenchymal involvement (Figure 1) indicating COVID-19 pneumonia. He was classified as a patient with severe COVID-19 infection. RT-PCR was still positive after one week.
Neurological consultation was performed, and brain CT, sinus CT, brain MRI (magnetic resonance imaging), and brain MRA (magnetic resonance angiography) were ordered. The sinus CT revealed extensive opacification of right ethmoid, maxillary sinus, and nasal septum. There was also obstruction of right ostiomeatal complex (OMC) (Figure 2). MRI and MRA showed normal brain tissue (not shown).

To diagnose meningitis, lumbar puncture (LP) was done and cerebrospinal fluid (CSF) was collected for culture. The CSF culture was negative for any bacterial infection. However, cytology examination showed acute inflammation in the CSF sample.

The clinical and radiographic findings were highly suspicious for acute invasive fungal rhinosinusitis with orbital involvement. Thus, debridement of the sinuses was done and the specimen was sent for culture and histopathology examination. On histopathology examination, broad aseptate filamentous fungal hyphae were seen and therefore mucormycosis infection was confirmed.

\subsection{Treatment}

For COVID-19 infection, treatment was initiated with IV remdesivir (loading dose: $200 \mathrm{mg}$ followed by $100 \mathrm{mg}$ /day after each hemodialysis session for 10 days), dexamethasone (16 mg twice a day), ivermectin (12 mg STAT), IV $\mathrm{N}$-acetyl cysteine (NAC) infusion (2 $\mathrm{g}$ twice a day), and colchicine $(0.5 \mathrm{mg}$ twice a day). Oxygen therapy was done using an oxygen mask with reservoir bags (OMR) and oxygen saturation level improved to $88 \%$ on day 6 of hospitalization.

Insulin glargine (30 units in the morning and 16 units at night) and insulin aspart (18, 18, 16 units before each meal) were prescribed to control diabetes.

Amlodipine $5 \mathrm{mg}$ per day and prazosin $0.25 \mathrm{mg}$ three times a day were administered for hypertension treatment.

Liposomal amphotericin B was started at a dose of $5 \mathrm{mg} / \mathrm{kg} /$ day to treat mucormycosis infection.

\section{3 | DISCUSSION}

There are limited data about the effect of COVID-19 on mucormycosis or other fungal infections. On the one hand, the massive inflammatory immune response following COVID-19 and the administration of immunosuppressive drugs provides a favorable condition for the development of fungal co-infections. It is suggested that venous thromboembolism (VTE) caused by COVID-19 infection leads to mechanical ventilation, central venous catheterization, and surgery, all of which could cause damage to the 
elastic lamina of the blood vessels and eventually trigger the propagation of mucorales. ${ }^{3}$ Furthermore, comorbidities such as diabetes mellitus and diabetic nephropathy are risk factors for both COVID-19 and mucormycosis. ${ }^{8}$

TA B LE 1 Ancillary tests during patient hospitalization

\begin{tabular}{|c|c|c|}
\hline $\begin{array}{l}\text { Blood plasma/ } \\
\text { serum }\end{array}$ & result & $\begin{array}{l}\text { Reference } \\
\text { serum (normal) }\end{array}$ \\
\hline Sodium & $140 \mathrm{mEq} / \mathrm{L}$ & $135-145 \mathrm{mEq} / \mathrm{L}$ \\
\hline Potassium & $4.1 \mathrm{mEq} / \mathrm{L}$ & $3.5-5.0 \mathrm{mEq} / \mathrm{L}$ \\
\hline $\mathrm{BS}$ & 291 mg/dl (high) & $70-140 \mathrm{mg} / \mathrm{dl}$ \\
\hline FBS & 186 mg/dl(high) & $70-110 \mathrm{mg} / \mathrm{dl}$ \\
\hline Creatinine & 5.4 mg/dl (high) & $0.5-1.5 \mathrm{mg} / \mathrm{dl}$ \\
\hline BUN & $46.7 \mathrm{mg} / \mathrm{dl}$ (high) & $7-20 \mathrm{mg} / \mathrm{dl}$ \\
\hline Magnesium & 3.8 mEq/L(high) & $1.8-2.6 \mathrm{mEq} / \mathrm{L}$ \\
\hline CRP & $89.1 \mathrm{mg} / \mathrm{l}$ (high) & Less than $6 \mathrm{mg} / \mathrm{L}$ \\
\hline SGOT & $189 \mathrm{U} / \mathrm{L}$ (high) & Less than $40 \mathrm{U} / \mathrm{L}$ \\
\hline SGPT & 61 U/L (high) & Less than $40 \mathrm{U} / \mathrm{L}$ \\
\hline $\begin{array}{l}\text { Alkaline } \\
\text { phosphatase }\end{array}$ & $219 \mathrm{U} / \mathrm{L}$ & 80-306 U/L \\
\hline phosphorus & 5.3 mg/dl (high) & 3.4 to $4.5 \mathrm{mg} / \mathrm{dl}$ \\
\hline Calcium & $8.6 \mathrm{mg} / \mathrm{dl}$ & $8.5-10.5 \mathrm{mg} / \mathrm{dl}$ \\
\hline Bicarbonate & $17.8 \mathrm{mEq} / \mathrm{L}(\mathrm{low})$ & $22-26 \mathrm{mEq} / \mathrm{L}$ \\
\hline $\mathrm{PCO} 2$ & $41.7 \mathrm{~mm} \mathrm{Hg}$ & $35-40 \mathrm{~mm} \mathrm{Hg}$ \\
\hline PH & 7.23 & $7.31-7.41$ \\
\hline $\mathrm{LDH}$ & 580 U/L (high) & $140-280 \mathrm{U} / \mathrm{L}$ \\
\hline D-Dimer & positive & Negative \\
\hline WBC & $11,300 \mathrm{~mm}^{3}$ (high) & $4000-10,000 / \mathrm{mm}^{3}$ \\
\hline $\mathrm{R} \mathrm{B} \mathrm{C}$ & $4.65 \mathrm{mill} / / \mathrm{mm}^{3}$ & $4.5-6.5 / \mathrm{mm}^{3}$ \\
\hline $\mathrm{Hb}$ & 11.5 (low) & $13.5-18 \mathrm{~g} / \mathrm{dl}$ \\
\hline Hct & $36.0 \%$ & $42-52 \%$ \\
\hline ESR & $39 \mathrm{~mm} / \mathrm{hr}$ & Less than 10 \\
\hline Platelet & $17,0000 / \mathrm{mm}^{3}$ & $\begin{array}{l}150,000- \\
450,000 \mathrm{~mm}^{3}\end{array}$ \\
\hline
\end{tabular}

Abbreviations: BS, blood sugar; BUN, blood urea nitrogen; CRP, c-reactive protein; ESR, erythrocyte sedimentation rate; FBS, fast blood sugar; $\mathrm{Hb}$, hemoglobin; Hct, hematocrit; LDH, lactate dehydrogenase; SGOT, serum glutamic-oxaloacetic transaminase; SGPT, serum glutamic-pyruvic transaminase.
This case report is consistent with other case reports indicating the role of diabetes mellitus as a predisposing factor for SARS-CoV-2 and mucormycosis co-infection. Besides, this case had multiple underlying health conditions including diabetic ketoacidosis, diabetic nephropathy, hypertension, and a history of CABG, all of which increase COVID-19 severity and the risk of coinfection or superinfection with other microorganisms. ${ }^{9}$ Unfortunately, the patient had a poor prognosis due to extensive rhino-orbital mucormycosis and ARDS and died after one month of hospitalization.

Based on an extensive literature search, the presented case had some unique features compared to similar case reports. First of all, diabetic nephropathy followed by hemodialysis has not been reported in concurrent infection of COVID-19 and mucormycosis. Second, diabetic ketoacidosis as a comorbidity in SARS-CoV-2 co-infected with mucormycosis is reported in a limited number of case reports. ${ }^{10,11}$ The presented case was the only case with uncontrolled diabetes mellitus, diabetic ketoacidosis, diabetic nephropathy required hemodialysis, and hypertension who developed rhino-orbital mucormycosis following a severe COVID-19 infection. It should be noted that other underlying health conditions have also been associated with mucormycosis following COVID-19 such as hematological malignancies, hypothyroidism, asthma, and obesity in participants with a median age of 53.4 and male sex comprising the majority of the cases. ${ }^{12-19}$

As of July 4, 2021, four cases of rhino-orbital-cerebral mucormycosis following COVID-19 with a median age of 51.2 years have been reported in Iran, three of whom were women and one died. Diabetes mellitus was the most common underling condition (3 of 4). Amphotericin B was prescribed for all of them as the first line of treatment for mucormycosis infection. ${ }^{20-22}$

Mucormycosis is a serious opportunistic fungal infection that could lead to rhino-orbital, gastrointestinal, pulmonary, renal, cutaneous, and disseminated infection. The infection enters the host via the respiratory tract. Since it has a noticeable affinity for arteries, it grows in the internal elastic lamina, which triggers thrombosis and
FIG URE 1 Computed tomography showing bilateral peripheral ground-glass opacification (GGO) and thickness in the interalveolar septum with extensive lung parenchymal involvement in the corresponding lung window (A) and mediastinal sections (B)
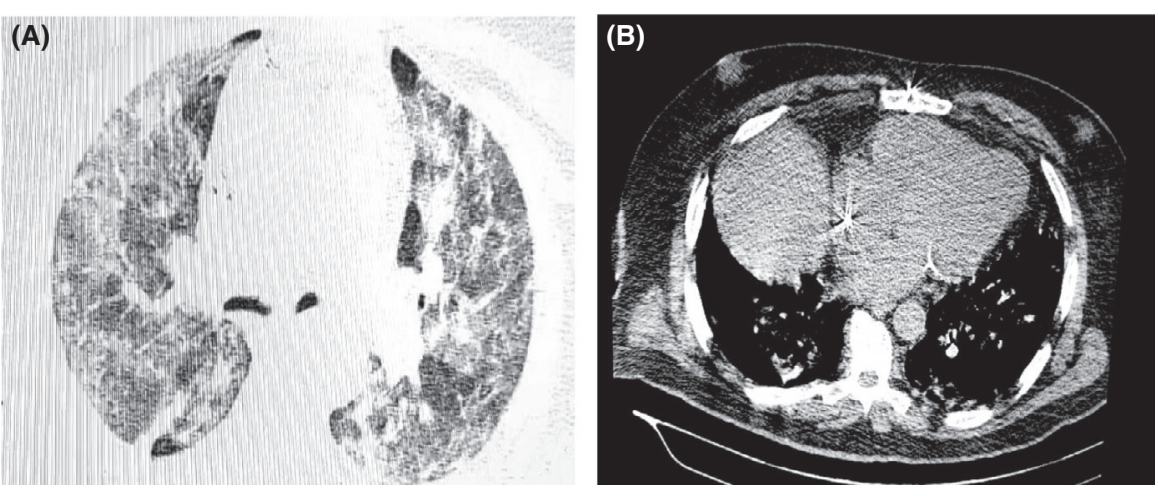

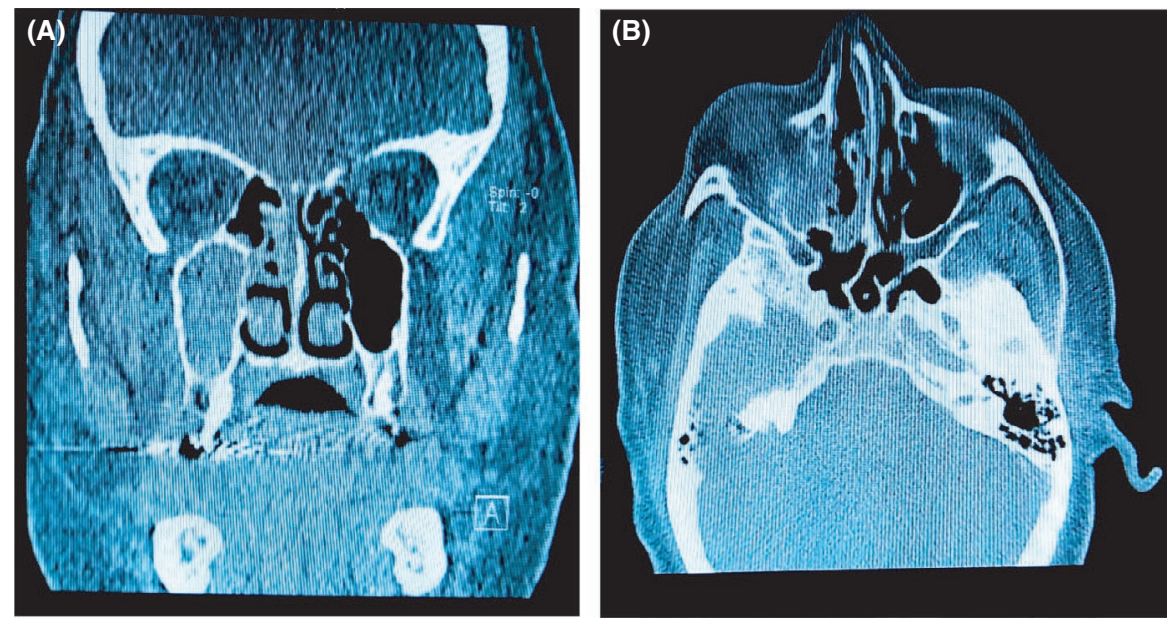

FIG URE 2 Non-contrast computed tomography showing mucoperiosteal thickening in the right maxillary, ethmoid sinus, and blocked right ostiomeatal complex (OMC). Coronal section (A) axial sections (B) infarction. ${ }^{23}$ The disease can progress from the nose and sinuses directly or through vascular occlusion. Invasion through the superior orbital fissure, ophthalmic vessels, cribriform plate, carotid artery, or possibly via the perineural route can involve the intracranial components. ${ }^{24}$

Surgical debridement of the necrotic tissue, treatment with liposomal amphotericin B as an antifungal therapy, and control of risk factors are suggested to improve survival. ${ }^{25}$

Since COVID-19 pandemic is continuing, attention should be paid to co-infection of SARS-CoV-2 with other microorganisms. Administration of immunosuppressive drugs for COVID-19 patients with underlying health conditions should be done cautiously with continuous monitoring. In spite of extensive research into COVID-19 management, more studies are still required regarding the prevention and management of opportunistic infections in COVID-19 patients.

\section{CONCLUSION}

We presented a case of SARS-CoV-2 and mucormycosis co-infection with multiple underlying health conditions. Unfortunately, the patient died despite surgical debridement and antifungal and antiviral treatment. We believe that uncontrolled diabetes milieus made the patient susceptible to both severe COVID-19 and mucormycosis. Prevention and management guidelines as well as prophylactic treatment protocols are needed for similar complicated patients.

\section{ACKNOWLEDGMENTS}

The authors would like to thank Dr. Kaveh, Dr. Rouhani in Infectious Disease Section of Valiasr Naja Hospital, Tehran, Iran, for their contribution to this research, and the patient and his family for their patience and co-operation.

\section{CONFLICTS OF INTEREST}

The authors declare that there are no conflicts of interests.

\section{AUTHOR CONTRIBUTIONS}

$\mathrm{ZH}$ involved in concept, investigation, and manuscript preparation. MS and SE involved in the clinical case and data collection. SZ involved in supportive role in manuscript preparation and revision. NZSH reviewed the paper critically and comments were included. All authors approved the final version of the manuscript before submission.

\section{ETHICAL APPROVAL}

The study was conducted in accordance with the Declaration of Helsinki and national and institutional standards, and it was approved by Valiasr Naja Hospital medical ethics committee. Patient signed written informed consents and accepted the publication of clinical data for research.

\section{CONSENT}

Patient signed written informed consents and accepted the publication of clinical data for research.

\section{DATA AVAILABILITY STATEMENT}

The data that support the findings of this study are available from the corresponding author upon reasonable request.

\section{ORCID}

Zahra Heydarifard (10) https://orcid. org/0000-0002-2093-819X

\section{REFERENCES}

1. Dong E, Du H, Gardner L. An interactive web-based dashboard to track COVID-19 in real time. Lancet Infect Dis. 2020;20(5):533-534. 
2. Elezkurtaj S, Greuel S, Ihlow J, et al. Causes of death and comorbidities in hospitalized patients with COVID-19. Sci Rep. 2021;11(1):1-9.

3. Pandiar D, Kumar NS, Anand R, Kamboj M, Narwal A, Shameena P. Does COVID 19 generate a milieu for propagation of mucormycosis? Med Hypotheses. 2021;152:110613.

4. Eucker J, Sezer O, Graf B, Possinger K. Mucormycoses. Mycoses. 2001;44(7-8):253-260.

5. Dolatabadi S, Ahmadi B, Rezaei-Matehkolaei A, et al. Mucormycosis in Iran: a six-year retrospective experience. $J$ Mycol Med. 2018;28(2):269-273.

6. Ribes JA, Vanover-Sams CL, Baker DJ. Zygomycetes in human disease. Clin Microbiol Rev. 2000;13(2):236-301.

7. Ibrahim AS, Spellberg B, Walsh TJ, Kontoyiannis DP. Pathogenesis of mucormycosis. Clin Infect Dis. 2012;54(suppl_1):S16-S22.

8. John TM, Jacob CN, Kontoyiannis DP. When uncontrolled diabetes mellitus and severe COVID-19 converge: the perfect storm for mucormycosis. J Fungi. 2021;7(4):298.

9. Singh AK, Singh R, Joshi SR, Misra A. Mucormycosis in COVID-19: a systematic review of cases reported worldwide and in India. Diabetes Metab Synd. 2021;15(4):102146.

10. Alekseyev K, Didenko L, Chaudhry B. Rhinocerebral mucormycosis and COVID-19 pneumonia. J Med Cases. 2021;12(3):85.

11. Waizel-Haiat S, Guerrero-Paz JA, Sanchez-Hurtado L, CallejaAlarcon S, Romero-Gutierrez L. A case of fatal rhino-orbital mucormycosis associated with new onset diabetic ketoacidosis and COVID-19. Cureus. 2021;13(2).):e13163.

12. Hanley B, Naresh KN, Roufosse C, et al. Histopathological findings and viral tropism in UK patients with severe fatal COVID-19: a post-mortem study. Lancet Microbe. 2020;1(6):e24 5-e253.

13. Mehta S, Pandey A. Rhino-orbital mucormycosis associated with COVID-19. Cureus. 2020;12(9).e10726.

14. Monte Junior ESD, Santos MELD, Ribeiro IB, et al. Rare and fatal gastrointestinal mucormycosis (zygomycosis) in a COVID-19 patient: a case report. Clin Endoscopy. 2020;53(6):746-749.

15. Placik DA, Taylor WL, Wnuk NM. Bronchopleural fistula development in the setting of novel therapies for acute respiratory distress syndrome in SARS-CoV-2 pneumonia. Radiol Case Rep. 2020;15(11):2378-2381.

16. Werthman-Ehrenreich A. Mucormycosis with orbital compartment syndrome in a patient with COVID-19. Am J Emerg Med. 2021;42:264.e5-264.e8.
17. Mekonnen ZK, Ashraf DC, Jankowski T, et al. Acute invasive rhino-orbital mucormycosis in a patient with COVID-19associated acute respiratory distress syndrome. Ophthalmic Plast Reconstr Surg. 2021;37(2):e40.

18. Pasero D, Sanna S, Liperi C, et al. A challenging complication following SARS-CoV-2 infection: a case of pulmonary mucormycosis. Infection. 2021;49(5):1055-1060.

19. Zurl C, Hoenigl M, Schulz E, et al. Autopsy proven pulmonary mucormycosis due to Rhizopus microsporus in a critically ill COVID-19 patient with underlying hematological malignancy. J Fungi. 2021;7(2):88.

20. Veisi A, Bagheri A, Eshaghi M, Rikhtehgar MH, Rezaei Kanavi M, Farjad R. Rhino-orbital mucormycosis during steroid therapy in COVID-19 patients: a case report. Eur J Ophthalmol. 2021;112067212110094.

21. Karimi-Galougahi M, Arastou S, Haseli S. Fulminant mucormycosis complicating coronavirus disease 2019 (COVID-19). Int Forum Allergy Rhinol. 2021;11(6):1029-1030.

22. Tabarsi P, Khalili N, Pourabdollah M, Sharifynia S, Naeini AS, Ghorbani J, et al. COVID-19 associated rhinosinusitis mucormycosis due to Rhizopus oryzae: a rare but potentially fatal infection occurring after treatment with corticosteroids. 2021.

23. Gupta S, Goyal R, Kaore NM. Rhino-orbital-cerebral mucormycosis: battle with the deadly enemy. Indian J Otolaryngol Head Neck Surg. 2020;72(1):104-111.

24. Bawankar P, Lahane S, Pathak P, Gonde P, Singh A. Central retinal artery occlusion as the presenting manifestation of invasive rhino-orbital-cerebral mucormycosis. Taiwan J Ophthalmol. 2020;10(1):62.

25. Hirabayashi KE, Idowu OO, Kalin-Hajdu E, et al. Invasive fungal sinusitis: risk factors for visual acuity outcomes and mortality. Ophthalmic Plast Reconstr Surg. 2019;35(6):535-542.

How to cite this article: Heydarifard Z, Safaei M, Zadheidar S, Ehsan S, Shafiei-Jandaghi NZ. Mucormycosis infection in severe COVID-19 patient with multiple underlying health conditions. Clin Case Rep. 2021;9:e05009. https://doi. org/10.1002/ccr3.5009 